\title{
Uso de ciclosporina $0,05 \%$ tópica no tratamento do olho seco de pacientes portadores do vírus HIV
}

\section{Topical cyclosporine $0.05 \%$ for the treatment of dry eye disease in patients infected with the human immunodeficiency virus}

Rodrigo de Pinho Paes Barreto', Ana Luiza Biancardi², Emília Matos Nascimento ${ }^{3}$, Basílio de Bragança Pereira ${ }^{4}$, Haroldo Vieira de Moraes $\mathrm{Jr}^{5}$

RESUMO
Objetivo: O presente estudo comparou a eficácia do tratamento da síndrome do olho
seco em pacientes infectados pelo HIV com suplementação lacrimal
(carboximetilcelulose sódica $0,5 \%$ ) ou com suplementação lacrimal associada à tera-
pia anti-inflamatória (carboximetilcelulose sódica 0,5\% e ciclosporina $0,05 \%$ ). Méto-
dos: Vinte pacientes portadores do vírus HIV provenientes do ambulatório de
Infectologia do Hospital Universitário Clementino Fraga Filho da Universidade Fede-
ral do Rio de Janeiro foram selecionados. O diagnóstico de síndrome do olho seco foi
baseado no questionário para olho seco (Ocular Surface Disease Index - OSDI ${ }^{\circledR}$ ), teste
de Schirmer I, tempo de ruptura do filme lacrimal e coloração da superfície ocular com
rosa bengala a $1 \%$. Os pacientes foram distribuídos em dois grupos com dez pacientes
(20 olhos) e acompanhados durante seis meses. O grupo I foi tratado com colírio de
carboximetilcelulose sódica 0,5\% e o grupo II foi tratado com a associação de
carboximetilcelulose sódica 0,5\% e ciclosporina 0,05\% tópica. Resultados: Comparan-
do os resultados no início e no final do tratamento, a associação com ciclosporina 0,05\%
mostrou-se superior (p<0,05) para o teste de Schirmer I. Conclusão: O presente estudo
sugere que o uso de ciclosporina $0,05 \%$ tópica melhora a produção lacrimal em pacien-
tes infectados pelo HIV,apresentando um importante papel como adjuvante no trata-
mento do olho seco nestes pacientes.
ClinicalTrials.gov Identifier: NCT00797030

Descritores: Síndrome do olho seco/quimioterapia; Ceratoconjuntivite seca/ quimioterapia, Ciclosporina/uso terapêutico; Lágrimas artificiais/administração \& dosagem; Administração tópica; Soropositividade para HIV

\footnotetext{
'Mestre em Oftalmologia pela Universidade Federal do Rio de Janeiro - UFRJ - Rio de Janeiro (RJ), Brasil; ${ }^{2}$ Mestre em Oftalmologia pela Universidade Federal do Rio de Janeiro - UFRJ - Rio de Janeiro (RJ), Brasil;

3Pós-Graduanda nível Doutorado em Bioestatística da Universidade Federal do Rio de Janeiro - UFRJ - Rio de Janeiro (RJ), Brasil; ${ }^{4}$ Professor Titular do Departamento de Bioestatística da Universidade Federal do Rio de Janeiro - UFRJ - Rio de Janeiro (RJ), Brasil; Professor Associado do Departamento de Oftalmologia da Universidade Federal do Rio de Janeiro - UFRJ - Rio de Janeiro (RJ), Brasil.

Trabalho realizado no Hospital Universitário Clementino Fraga Filho - Universidade Federal do Rio de Janeiro - UFRJ - Rio de Janeiro (RJ), Brasil.
} 


\section{INTRODUÇÃO}

A síndrome do olho seco se refere a uma doença da superfície ocular com diversas etiologias que frequentemente coexistem. A prevalência precisa da síndrome do olho seco é difícil pela falta de critérios diagnósticos concisos e pela subjetividade dos sintomas ${ }^{(1)}$. Estudos prévios estimaram prevalências compreendidas entre $5,5 \%$ e $33,7 \%{ }^{(2-10)}$.

Em 1995, encontros realizados no National Eye Institute (NEI) propuseram que o olho seco pode ser decorrente de uma deficiência da produção aquosa lacrimal ou de uma evaporação excessiva, com dano à superfície ocular interpalpebral e sintomas oculares ${ }^{(11)}$.

Em 2003, a classificação tripla de Madri propôs que o diagnóstico do olho seco fosse baseado em três parâmetros: etiologia, histopatologia e gravidade clínica ${ }^{(12)}$.

Em 2006, o painel Delphi propôs um consenso para classificação e tratamento do olho seco. Um novo termo para a síndrome do olho seco foi sugerido: síndrome de disfunção lacrimal. ${ }^{(13)}$

Em 2007, foram publicados os resultados do International Dry Eye Workshop (DEWS $)^{(14)}$,com o objetivo de ampliar os conceitos estabelecidos pelo NEI (1995) ${ }^{(11)}$. O olho seco foi definido como uma doença multifatorial da lágrima e da superfície ocular, resultando em desconforto, turvação visual e instabilidade do filme lacrimal, com dano potencial à superfície ocular. É associado ao aumento da osmolaridade da lágrima e à inflamação da superfície ocular.

O tratamento do olho seco deve levar em consideração a etiologia e a gravidade da doença. Medidas gerais para evitar a evaporação da lágrima, como evitar regiões secas e o uso de ar condicionado, são úteis, porém insuficientes. A terapêutica atual envolve, além de medidas comportamentais, a suplementação e retenção lacrimal, substitutos biológicos da lágrima, terapia anti-inflamatória e o uso de ácidos graxos essenciais ${ }^{(15)}$.

As alterações oculares em pacientes infectados pelo HIV são comuns, afetando $70 \%$ a $80 \%$ destes indivíduos em algum ponto no curso da doença ${ }^{(16,17)}$. Um estudo ${ }^{(18)}$ avaliou os principais sintomas da infecção pelo HIV em 4042 adultos. As queixas oftalmológicas, citadas por 32,4\% dos pacientes, ficaram em oitavo lugar, o que reforça a importância do acompanhamento oftalmológico de indivíduos com este vírus.

A etiologia do olho seco associada ao vírus HIV ainda não é bem estabelecida. A redução lacrimal pode estar associada à infiltração linfocítica e eventual destruição dos ácinos e ductos da glândula lacrimal ${ }^{(19)}$. A prevalência do olho seco em pacientes infectados pelo HIV em estudos prévios variou de $7,79 \%$ a $38,8 \%$ e a sintomatologia do olho seco tem um impacto relevante na qualidade de vida destes pacientes ${ }^{(2024)}$.

O presente estudo avaliou 20 pacientes portadores do vírus HIV com diagnóstico de olho seco e comparou a eficácia do tratamento com suplementação lacrimal (carboximetilcelulose sódica $0,5 \%$ ) ou com suplementação lacrimal associada à terapia anti-inflamatória (carboximetilcelulose sódica 0,5\% e ciclosporina $0,05 \%$ ).

\section{Métodos}

Foi realizado um estudo prospectivo clínico randomizado de pacientes infectados pelo vírus da imunodeficiência humana (HIV) portadores de olho seco. Noventa e seis pacientes responderam ao questionário Ocular Surface Disease Index (OSDI $\left.{ }^{\circledR}\right)$ e os que obtiveram pontuação maior que 25 e preencheram os critérios de inclusão e exclusão foram selecionados para exame oftalmológico completo e pesquisa de sinais de olho seco através dos seguintes testes: Tempo de ruptura do filme lacrimal (TRFL), teste de Schirmer I e coloração da superfície ocular por rosa bengala $1 \%$. A realização dos testes foi rigorosamente semelhante para evitar fatores de_confundimento.

O TRFL foi realizado instilando-se uma gota de fluoresceína sódica a 2\% (Ophthalmos ${ }^{\circledR}$, São Paulo, Brasil) no fundo de saco conjuntival inferior e solicitando ao paciente que piscasse várias vezes para, então, permanecer com o olho aberto. O observador, utilizando luz de cobalto na lâmpada de fenda, contou o tempo da abertura do olho até o aparecimento da mancha seca. Foi considerado compatível com olho seco quando menor ou igual a dez segundos ${ }^{(25)}$.

A coloração da superfície ocular por rosa bengala foi realizada instilando-se uma gota de colírio rosa bengala a $1 \%$ (Ophthalmos ${ }^{\oplus}$, São Paulo, Brasil) no fundo de saco conjuntival inferior e foi observado o tingimento conjuntival. Foi considerado compatível com olho seco quando ocorreu pontuação maior que três, segundo a escala de Van Bijsterveld ${ }^{(26)}$.

O teste de Schirmer I foi realizado com tiras de papel filtro estéreis padronizadas (Ophthalmos ${ }^{\circledR}$, São Paulo, Brasil), em que uma das extremidades foi dobrada na altura de cinco $\mathrm{mm}$ e inserida no fundo de saco conjuntival, na união do terço externo e médio da pálpebra inferior. Após 5 minutos, o papel filtro foi retirado e depois de 1 minuto de espera, foi medido o quanto ele se 
umidificou. Foi considerado sugestivo de olho seco quando menor ou igual a $10 \mathrm{~mm}^{(27)}$.

Foram incluídos 20 indivíduos infectados pelo vírus HIV, na faixa etária entre 18 e 45 anos que apresentaram sinais e sintomas de olho seco moderado. Foram excluídos os indivíduos que apresentassem outras condições associadas ao olho seco: coinfecção (vírus da hepatite $\mathrm{B}$ e C), mulheres na menopausa, portadores de doenças reumáticas e/ou em uso de medicações indutoras de olho seco (diuréticos, anti-histamínicos, betabloqueadores, antidepressivos, ansiolíticos), usuários de lente de contato, utilização de colírios beta bloqueadores e blefarite.

Os pacientes foram distribuídos aleatoriamente em dois grupos com 20 olhos cada e acompanhados durante seis meses. O grupo I foi tratado com colírio de carboximetilcelulose sódica $0,5 \%$ instilado quatro vezes ao dia, e o grupo II foi tratado com a associação de carboximetilcelulose sódica $0,5 \%$ (quatro vezes ao dia) e ciclosporina $0,05 \%$ tópica (duas vezes ao dia). Nas visitas, os pacientes responderam ao questionário OSDI ${ }^{\circledR}$ e foram submetidos ao TRFL, teste de Schirmer I e coloração por rosa bengala a $1 \%$.

Foi comparada a eficácia do tratamento nos dois grupos através da curva de sobrevivência de KaplanMeyer e do teste Tarone-Ware, para as variáveis TRFL, Rosa Bengala e Schirmer I. Para comparar o comportamento dos grupos em relação ao OSDI ${ }^{\varpi}$, os valores obtidos do questionário foram inicialmente testados para verificar a normalidade dos dados através do teste w/s e posteriormente verificados a diferença entre as variâncias usando o teste $\mathrm{F}$, aplicando-se então o teste $\mathrm{t}$ para analisar a diferença de médias.

\section{Resultados}

A mediana de idade dos pacientes foi de 41 (32 a 45 anos), 11 (55\%) eram mulheres, 9 (45\%) eram homens, o menor tempo de diagnóstico de infecção pelo HIV foi 8 meses e o maior foi 20 anos. Os grupos I e II, formados por 10 pacientes cada, se mostraram homogêneos em relação ao sexo, idade e condições clínicas, satisfatoriamente controlados através da terapia HAART. $(\mathrm{OSDI})^{\oplus}$

Pontuação no Ocular Surface Disease Índex

Os valores mensurados pelo questionário OSDI ${ }^{\oplus}$ foram submetidos ao teste de normalidade w/s, no qual se obteve no tempo zero w/s 3.07 para o grupo I e 3.17 para o grupo II e no tempo $180 \mathrm{w} / \mathrm{s} 2.17$ para o grupo I e 3.05 para o grupo II. Em geral, os dados parecem seguir uma distribuição normal.

O F-teste para comparar duas variâncias apresentou um p-valor de 0.44 para o tempo zero e p-valor de 0.78 para o tempo 180 .

$\mathrm{O}$ t-teste para as duas amostras apresentou p-valor de 0.06 para o tempo zero e p-valor de 0.17 para o tempo 180.

Concluindo, não há significância estatística em relação aos grupos estudados com relação à variável OSDI ${ }^{\oplus}$.

Tempo de ruptura do filme lacrimal

As curvas de sobrevivência de Kaplan-Meyer (Gráfico 1) indicam que os grupos não apresentaram diferença significativa entre si no decorrer dos 180 dias de tratamento, apesar do prolongamento do gráfico que representa um aumento no tempo de ruptura do filme lacrimal.

Coloração por rosa bengala

As curvas de sobrevivência de Kaplan-Meyer (Gráfico 2) indicam que os grupos não apresentaram diferença significativa entre si no decorrer dos 180 dias de tratamento, apesar da redução do gráfico que representa uma diminuição da pontuação da coloração da superfície ocular por rosa bengala.

Teste de Schirmer I

As curvas de sobrevivência de Kaplan-Meyer (Gráfico 3) indicam diferença estatisticamente significantes entre os dois grupos. Observa-se um prolongamento da curva contínua e uma manutenção da curva tracejada, o que representa uma maior produção lacrimal pelo grupo II.

\section{Discussão}

O olho seco é uma doença multifatorial da lágrima com dano potencial à superfície ocular, que resulta em desconforto, turvação visual e instabilidade do filme lacrimal ${ }^{(14)}$, com significativo impacto na qualidade de vida ${ }^{(28,29)}$.

Em nossa casuística, os grupos se mostraram homogêneos em relação ao sexo dos pacientes. Estudos prévios apresentaram resultados controversos em relação à prevalência da síndrome do olho seco em homens e mulheres portadores de HIV. Foram descritas prevalências iguais a $21 \%{ }^{(24)}$ e $38.8 \%{ }^{(21)}$ em homens e $16.9 \%{ }^{(23)}$ em mulheres. Rodrigues et al. ${ }^{(30)}$ relataram prevalência igual a $25,8 \%$, sem diferença em relação ao sexo. Na população geral, o sexo feminino é considerado um fator de risco consistente para desenvolvimento de olho seco ${ }^{(3,28)}$. Estas diferentes prevalências podem estar relacionadas aos critérios diagnósticos para olho seco 


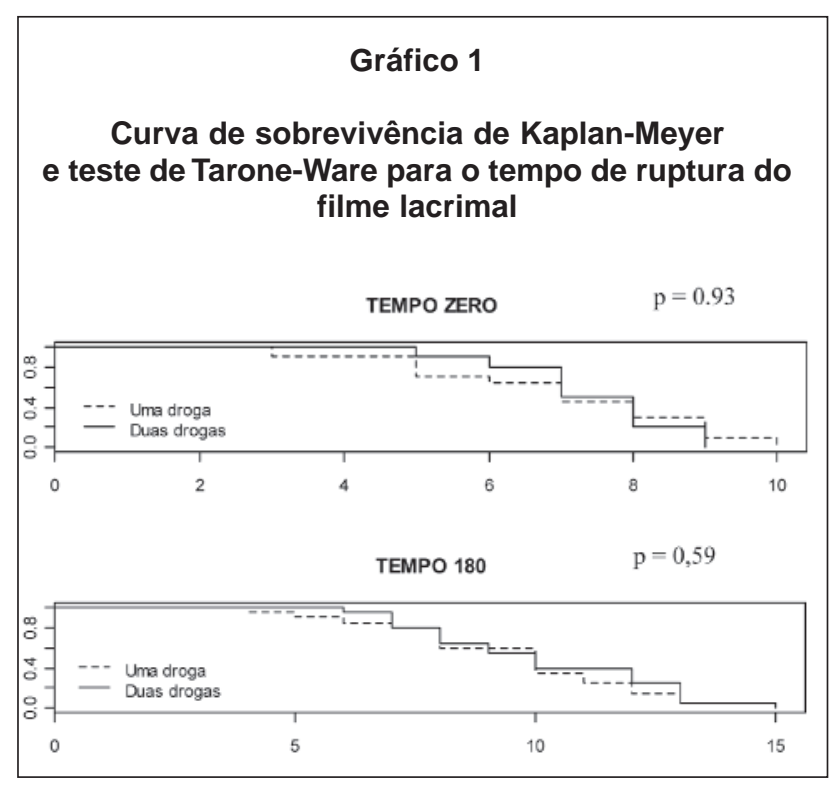

utilizados, ao número de pacientes avaliados e à idade dos pacientes.

No presente estudo, foram excluídos pacientes com idade superior a 45 anos. A idade é um fator de risco bem definido para olho seco ${ }^{(12,28)}$. A secreção lacrimal começa a declinar por volta dos 30 anos; entretanto, a produção excede a necessidade basal e não há sintomatologia. O nível crítico é atingido por volta de 45 anos, quando, em algumas circunstâncias, há sintomas relacionados ao olho seco. Aos 60 anos, a maioria das pessoas queixa-se de olho seco em algumas situações, tais como: exposição ao ar condicionado, uso de lentes de contato e à noite, quando há menor produção de lágrima devido à variação cicardiana ${ }^{(12)}$.

Em nossa casuística, os grupos se mostraram homogêneos em relação ao tempo de diagnóstico da infecção pelo HIV. Rodrigues e cols. ${ }^{(30)}$ observaram um aumento significativo da freqüência da síndrome de olho seco nos pacientes com doença acima de quatro anos, principalmente entre os que estavam em uso de terapia antirretroviral oral. No presente estudo, os pacientes apresentaram a doença controlada em uso de terapia HAART e os grupos eram homogêneos em relação às condições clínicas. Rodrigues e cols. ${ }^{(30)}$ não encontraram relação entre o decréscimo da produção lacrimal e a gravidade da infecção pelo vírus HIV. Geier et al. ${ }^{(22)}$ também não encontraram associação entre a contagem de CD4 ou a gravidade da infecção pelo HIV com a síndrome do olho seco.

Os principais estudos epidemiológicos ${ }^{(2-47-10)}$ sobre a síndrome do olho seco determinaram a prevalência de acordo com os sintomas referidos, tais como:

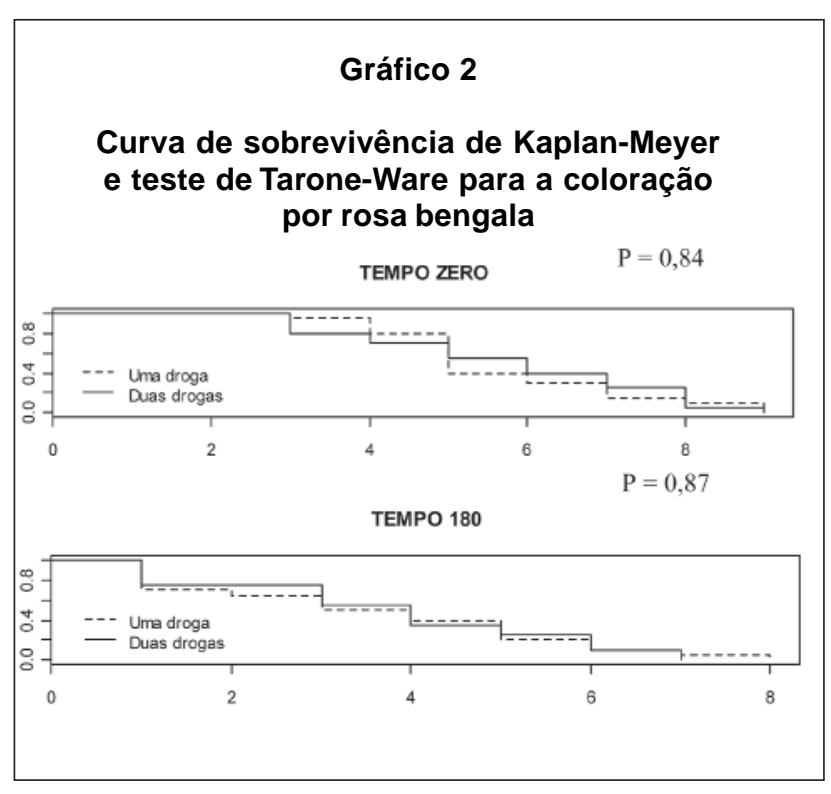

\section{Gráfico 3}

Curva de sobrevivência de Kaplan-Meyer e teste de Tarone-Ware para o teste de Schirmer I

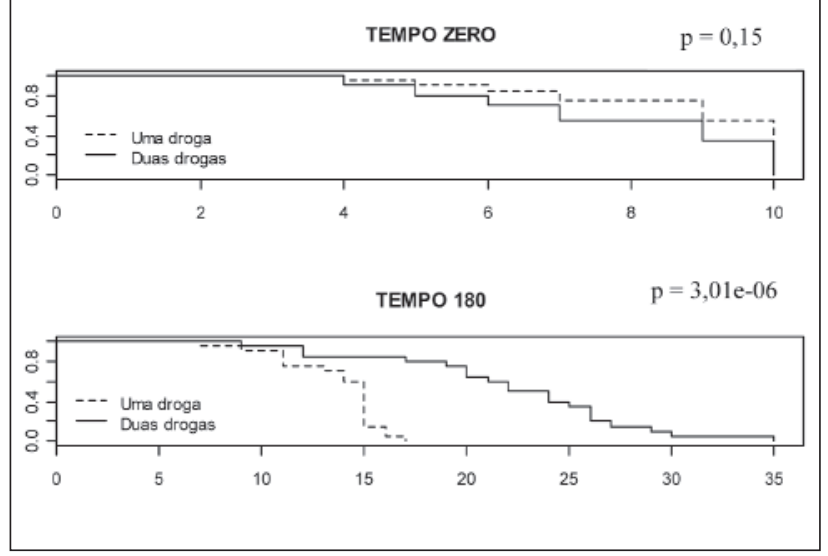

ressecamento, sensação de areia, queimação, hiperemia, olhos colados pela manhã, prurido e fotofobia. Tais sintomas são identificados através da aplicação de questionários como o OSDI ${ }^{\oplus}$.

Os questionários são empregados para rastreamento de indivíduos com olho seco, para verificar o impacto do tratamento nos sintomas ou para graduar a gravidade da doença ${ }^{(28)}$. O OSDI ${ }^{\circledR}$ é um questionário que avalia a gravidade do olho seco através de 12 perguntas que abordam sinais e sintomas, dificuldades visuais e fatores desencadeantes na semana anterior à avaliação. Este teste foi escolhido por ser rápido, confiável e de fácil entendimento, resultando em uma 
pontuação mesmo que o paciente não tenha respondido a todas as perguntas ${ }^{(31-33)}$.

O presente estudo avaliou subjetivamente os sintomas e objetivamente os sinais relacionados ao olho seco. Foram utilizados, além do questionário OSDI ${ }^{\circledast}$, três testes diagnósticos para olho seco (TRFL, teste de Schirmer I e coloração por rosa bengala a 1\%). Não existe um teste padrão-ouro para o diagnóstico de olho seco ${ }^{(34)}$. Entretanto, estes testes são bem estabelecidos na propedêutica desta síndrome. Segundo o painel Delphi ${ }^{(13)}$, os testes mais frequentemente utilizados pelos especialistas são a coloração por fluoresceína, o TRFL, o teste de Schirmer e a coloração por rosa bengala. Estudos prévios avaliaram a sensibilidade e especificidade destes testes. Vitali et al. ${ }^{(35)}$ encontraram sensibilidade igual a $83 \%$ e especificidade igual a $68 \%$ para o valor de referência de $10 \mathrm{~mm}$ em cinco minutos para o teste de Schirmer I. Os mesmos autores encontraram sensibilidade igual a $72 \%$ e especificidade igual a $62 \%$ para o valor de referência menor que 10 segundos para oTRFL. Goren et al. ${ }^{(36)}$ encontraram sensibilidade igual a $25 \%$ e especificidade igual a $90 \%$ para qualquer pontuação na coloração da superfície ocular com rosa bengala.

O objetivo do tratamento do olho seco é melhorar os sintomas e os sinais da superfície ocular e a escolha do tratamento deve considerar a etiologia e a gravidade da doença.

As lágrimas artificiais lubrificam e hidratam a superfície ocular através da substância ativa da sua composição. Entretanto, os conservantes utilizados para manter a lágrima artificial estéril podem danificar o epitélio dependendo da frequência de uso do colírio. ${ }^{(37,38)}$ No presente estudo, foi utilizada a substância ativa carboximetilcelulose sódica na concentração de $0,5 \%$ com o conservante clorito de sódio, que quando exposto à luz, se decompõe em íons cloreto e água, evitando o dano epitelial devido ao uso prolongado do colírio ${ }^{(37,38)}$.

Pacientes com olho seco possuem inflamação na superfície ocular e nas glândulas lacrimais mediadas por citocinas ${ }^{(14)}$. A ciclosporina é uma droga imunomoduladora que inibe a ativação das células $\mathrm{T}$, a produção destas citocinas inflamatórias e a cascata da apoptose ${ }^{(39)}$. A eficácia da ciclosporina no tratamento do olho seco já foi comprovada em estudos prévios ${ }^{(40-42)}$, porém não há relatos do seu uso no olho seco associado à infecção pelo HIV.

Com relação ao questionário OSDI ${ }^{\circledR}$, aplicado em todas as visitas, observa-se que nos dois grupos há redução da pontuação, o que indica melhora dos sintomas, todavia não ocorreu diferença estatisticamente significante entre os dois grupos ao final do tratamento. Em nossa casuística, embora o TRFL tenha aumentado nos dois grupos, não houve uma diferença estatisticamente significante do TRFL entre os grupos após 180 dias de tratamento.

Com relação à coloração da superfície ocular por rosa bengala, houve redução da pontuação de acordo com a escala de van Bijsterveld nos grupos I e II, porém a diferença entre os dois grupos não foi estatisticamente significante ao término do tratamento.

Com relação ao teste de Schirmer I, ocorreu um aumento dos valores após o tratamento nos dois grupos, entretanto o tratamento do grupo II mostrou-se superior ao tratamento do grupo I.

$\mathrm{O}$ efeito benéfico da ciclosporina na produção lacrimal é bem estabelecido. Quando a etiologia do olho seco relaciona-se à diminuição da produção aquosa e à inflamação da superfície ocular, sabe-se que a ciclosporina é bem indicada. Espera-se que o aumento da produção lacrimal resulte em redução da sintomatologia e dos sinais na superfície ocular. Entretanto, o grupo II não apresentou diferenças nos sintomas e nos outros sinais, apesar da melhora significativa do teste de Schirmer I em relação ao grupo I. Tal fato reforça o conceito de que o olho seco é uma doença multifatorial e ainda não completamente elucidada em pacientes HIV-positivos.

A literatura carece de estudos que avaliaram o tratamento de pacientes infectados pelo HIV com olho seco. O presente estudo é o primeiro trabalho que avaliou a eficácia do tratamento do olho seco em pacientes portadores do vírus HIV com suplementação lacrimal e terapia anti-inflamatória e sugere que o uso de ciclosporina tópica melhore a produção lacrimal nestes pacientes. Entretanto, é possível que o tratamento destes pacientes devesse envolver medidas adicionais. $\mathrm{O}$ papel do HIV na patogênese do olho seco deve ser mais bem investigado para direcionar melhor a terapêutica.

Algumas limitações deste estudo merecem considerações. Seria interessante realizar o estudo com um número maior de pacientes, para melhor avaliação do impacto do tratamento na sintomatologia e no exame oftalmológico dos portadores do vírus HIV e olho seco. Como o olho seco é uma doença multifatorial, qualquer outra condição associada ao olho seco foi considerada critério de exclusão, para evitar fatores de confundimento. Além disso, para aumentar a adesão ao tratamento, o presente estudo não incluiu pacientes com sintomas de olho seco leve. Tais critérios metodológicos reduziram a amostra utilizada. A exata fisiopatologia do olho seco em pacientes infectados pelo HIV ainda não é totalmente 
compreendida e a definição da síndrome do olho seco ainda é controversa, o que dificulta o diagnóstico desta doença complexa.

\section{Conclusão}

O uso de carboximetilcelulose sódica $0,5 \%$ foi tão eficaz quanto o uso da carboximetilcelulose sódica $0,5 \%$ associada à ciclosporina $0,05 \%$ na redução dos sintomas medidos pelo OSDI ${ }^{\oplus}$, assim como na melhora do TRFL e da coloração da superfície ocular por rosa bengala a $1 \%$ em pacientes infectados pelo HIV com olho seco.

Entretanto, comparativamente, o tratamento do olho seco com suplementação lacrimal (carboximetilcelulose sódica $0,5 \%$ ) associada à terapia anti-inflamatória (ciclosporina $0,05 \%$ tópica) mostrou-se superior ao uso isolado de carboximetilcelulose sódica $0,5 \%$ em relação à produção lacrimal, medida pelo teste de Schirmer I.

\section{Abstract}

Purpose: This study evaluates the use of topical cyclosporine $0.05 \%$ and sodium carboxymethylcellulose $0.5 \%$ for the treatment of dry eye disease in patients infected with the human immunodeficiency virus. Methods: Twenty HIV-positive-patients were selected from the Department of Infectious Diseases of the Federal University of Rio de Janeiro Hospital. Dry eye diagnosis was based on a dry eye questionnaire (Ocular Surface Disease Index - OSDI $I^{\oplus}$ ), Schirmer I test, break up time and $1 \%$ rose bengal staining of the ocular surface. The patients were divided into two groups with ten patients (20 eyes). Group I received sodium carboxymethylcellulose $0.5 \%$ drops and group II received sodium carboxymethylcellulose $0.5 \%$ drops and topical cyclosporine $0.05 \%$ for six months. Results: Comparing the results at the beginning and in the end of the treatment, the use of cyclosporine was superior $(p<0.05)$ for the Schirmer I test. Conclusion: This study suggests that topical cyclosporine improves lacrimal production and has an important role as an adjuvant therapy for dry eye disease in patients infected with the human immunodeficiency virus.

\section{ClinicalTrials.gov Identifier: NCT00797030}

Keywords: Dry eye syndromes/drug therapy; keratoconjuntivitis sicca/drug therapy, Cyclosporine/ therapeutic use; Ophthalmic solutions/administration \& dosage;Administration, topical; HIV seropositivity

\section{REFERÊNCIAS}

1. Johnson ME, Murphy PJ. Changes in the tear film and ocular surface from dry eye syndrome. Progress in retinal and eye research 2004;23(4):449-74.

2. McCarty CA, Bansal AK, Liv ingston PM, Stanislavsky YL, Taylor HR. The epidemiology of dry eye in Melbourne, Australia. Ophthalmology. 1998;105(6):1114-9.

3. Schaumberg DA, Sullivan DA, Buring JE, Dana MR. Prevalence of dry eye syndrome among US women. Am J Ophthalmol. 2003:136(2);318-26.

4. Moss SE, Klein R, Klein BE. Prevalence of and risk factors for dry eye syndrome. Arch Ophthalmol. 2000;118(9):1264-8.

5. Schein OD, Hochberg MC, Munoz B, Tielsch JM, BandeenRoche K, Provost T, et al. Dry eye and dry mouth in the elderly: a population-based assessment. Arch Intern Med. 1999;159(12):1359-63.

6. Schein OD, Munoz B, Tielsch JM, Bandeen-Roche K, West S. Prevalence of dry eye among the elderly. Am J Ophthalmol. 1997;124(6):723-8.

7. Munoz B, West SK, Rubin GS, Schein OD, Quigley HA, Bressler $\mathrm{SB}$, et al. Causes of blindness and visual impairment in a population of older Americans: The Salisbury Eye Evaluation Study. Arch Ophthalmol. 2000;118(6):819-25.

8. Chia EM, Mitchell P, Rochtchina E, Lee AJ, Maroun R, Wang JJ. Prevalence and associations of dry eye syndrome in an older population: the Blue Mountains Eye Study. Clin Experiment Ophthalmol. 2003;31(3):229-32.

9. Lee AJ, Lee J, Saw SM, Gazzard G, Koh D, Widjaja D, et al. Prevalence and risk factors associated with dry eye symptoms: a population based study in Indonesia. Br J Ophthalmol. 2002;86(12):1347-51.

10. Lin PY, Tsai SY, Cheng CY, Liu JH, Chou P, Hsu WM. Prevalence of dry eye among an elderly Chinese population in Taiwan: the Shihpai Eye Study. Ophthalmology. 2003;110(6):1096-101.

11. Lemp MA. Report of the National Eye Institute/Industry workshop on Clinical Trials in dry eyes. CLAO J. 1995;21(4):221-32.

12. Murube J, Benitez Del Castillo JM, Chenzhuo L, Berta A, Rolando M. Triple clasificación de Madrid para el ojo seco. Arch Soc Esp Oftalmol. 2003;78(11):587-93;595-601.

13. Behrens A, Doyle JJ, Stern L, Chuck RS, Mc Donnell PJ, Azar DT, et al. Dysfunctional tear syndrome: a Delphi approach to treatment recommendations. Cornea. 2006;25(8): 900- 7. 14 The definition and classification of dry eye disease: report of the definition and Classification Subcommittee of the International Dry Eye Workshop (2007). Ocul Surf 2007;5(2):75-92.

15. Management and therapy of dry eye disease: report of the Management and Therapy Subcommittee of the International Dry Eye Workshop (2007). Ocul Surf. 2007;5(2):163-78.

16. Cunningham ET Jr. Uveitis in HIV positive patients. Br J Ophthalmol 2000;84(3):233-7.

17. Cunningham ET Jr, Margolis TP. Ocular manifestations of HIV infection. N Engl J Med. 1998;339(4):236-44.

18. Mathews WC, McCutchan JA, Asch S, Turner BJ, Gifford AL, Kuromiya K, et al. National estimates of HIV-related symptom prevalence from the HIV cost and service utilization study. Med Care. 2000;38(7):750-62.

19. Pflugfelder SC, Wilhelmus KR, Osato MS, Matoba AY, Font RL. The autoimmune nature of aqueous tear deficiency. Ophthalmology 1986;93(12):1513-7. 
20. Kordossis T, Paikos S, Aroni K, Kitsanta P, Dimitrakopoulos A, Kavouklis E, et al. Prevalence of Sjögren-like syndrome in a cohort of HIV-1 positive patients: descriptive pathology and immunopathology. Br J Rheumatol. 1998:37(6):691-5.

21. DeCarlo DK, Penner SL, Schamerloh RJ, Fullard RJ. Dry eye among males infected with the human immunodeficiency virus. J Am Optom Assoc. 1995;66(9):533-8.

22. Geier SA, Libera S, Klauss V, Goebel FD. Sicca syndrome in patients infected with the human immunodeficiency virus. Ophthalmology. 1995;102(9):1319-24.

23. Lucca JA, Kung JS, Farris RL: Keratoconjunctivitis sicca in female patients infected with human immunodeficiency virus. CLAO J. 1994;20(1):49-51.

24. Lucca JA, Farris RL, Bielory L, Caputo AR Keratoconjunctivitis sicca in male patients infected with human immunodeficiency virus type 1. Ophthalmology.1990;9798):1008-10.

25. Yamane R, Yamane Y. Testes de secreção e drenagem lacrimal e estesiometria. In: Yamane R. Semiologia ocular. 2a ed. Rio de Janeiro: Cultura Médica; 2003 p.83-88

26. Van Bijsterveld OP. Diagnostic tests in the sicca syndrome Arch Ophthalmol. 1969;82(1):10-4.

27. Mattar DB, Kara-José N. Filme lacrimal. In: Belfort Jr, R, Kara-José, N. Córnea clínica - cirúrgica. São Paulo: Roca; 1996: 33-40.

28. The epidemiology of dry eye disease: report of the Epidemiology Subcommittee of the International Dry Eye WorkShop (2007). Ocul Surf. 2007;5(2):93-107.

29. Miljanovic B, Dana R, Sullivan DA, Schaumberg DA. Impact of dry eye syndrome on vision-related quality of life. Am J Ophthalmol. 2007;143(3):409-15.

30. Rodrigues ML, Rodrigues MLV, Holanda de Freitas JA. Estudo da síndrome de ceratoconjuntivite seca de pacientes soropositivos para o vírus da imunodeficiência adquirida humana tipo 1 e com síndrome da imunodeficiência adquirida, em uso ou não de terapia anti-retroviral combinada (HAART). Arq Bras Oftalmol. 2004;67(2):283-7.

31. Schiffman RM, Christianson MD, Jacobsen G, Hirsch JD, Reis BL. Reliability and validity of the Ocular Surface Disease Index. Arch Ophthalmol. 2000;118(5):615-21.

32. Ozcura F, Aydin S, Helvaci MR. Ocular Surface Disease Index for the diagnosis of dry eye syndrome. Ocul Immunol Inflam 2007;15(5):389-93.
33. Vitale S, Goodman LA, Reed GF, Smith JA. Comparison of the NEI-VFQ and OSDI questionnaires in patients with Sjogren's syndrome-related dry eye. Health Qual Life Outcomes. 2004;2:44.

34. Methodologies to diagnose and monitor dry eye disease: report of the Diagnostic Methodology Subcommittee of the International Dry Eye WorkShop (2007).Ocul Surf. 2007;5(2):108-52.

35. Vitali C, Moutsopoulos HM, Bombardieri S. The European Community Study Group on diagnostic criteria for Sjögren's syndrome. Sensitivity and specificity of tests for ocular and oral involvement in Sjögren's syndrome. Ann Rheum Dis. 1994;53(10):637-47.

36. Goren MB, Goren SB. Diagnostic tests in patients with symptoms of keratoconjunctivitis sicca in clinical practice. Am J Ophthalmol. 1988;106(5):570-4.

37. Management and therapy of dry eye disease: report of the Management and Therapy Subcommittee of the International Dry Eye WorkShop (2007). Ocul Surf. 2007;5(2):163-78.

38. Calonge M. The treatment of dry eye. Surv Ophthalmol. 2001;45 Suppl 2:227-39.

39. Nussenblatt RB, Palestine AG. Cyclosporin: immunology, pharmacology and therapeutic uses. Surv Ophthalmol. 1986;31(3):159-69.

40. Fullard RJ, Kaswan RM, Bounous DI, Hirsh SG. Tear protein profiles VS. Clinical characteristics of untreated and cyclosporine-treated canine KCS. J Am Optom Assoc. 1995;66(7):397-404.

41. Tsubota K, Saito I, Ishimaru N, Hayashi Y: Use of topical cyclosporine A in a primary Sjogrens syndrome mouse model. Invest Ophthalmol Vis Sci. 1998;39(9):1551-9.

42. Perry HD, Solomon R, Donnenfeld ED, Perry AR, Wittpenn JR, Greenman HE, et al. Evaluation of topical cyclosporine for the treatment of Dry Eye Disease. Arch Ophthalmol. 2008;126(8):1046-50.

\section{ENDEREÇO PARA CORRESPONDÊNCIA: \\ Rodrigo de Pinho Paes Barreto \\ Rua Vilhena de Moraes, $\mathbf{n}^{\circ} 100$ - bloco 02 - apt 903 \\ CEP 22793-140 - Barra da Tijuca - RJ \\ E-mail: barreto@live.com}

